\section{CORRECTION}

View Article Online

View Journal I View Issue
Check for updates

Cite this: J. Mater. Chem. A, 2020, 8 , 11058

DOI: 10.1039/d0ta90102d

rsc.li/materials-a

\title{
Correction: A disorder-free conformation boosts phonon and charge transfer in an electron- deficient-core-based non-fullerene acceptor
}

\author{
Chujun Zhang,,$^{+}$Jun Yuan,,$^{\mathrm{bc}}$ Ka Lok Chiu, ${ }^{\mathrm{a}}$ Hang Yin, ${ }^{\mathrm{a}}$ Weifang Liu, ${ }^{\mathrm{b}}$ \\ Guanhaojie Zheng, ${ }^{c}$ Johnny Ka Wai Ho, ${ }^{a}$ Zhenhuang Su, ${ }^{d}$ Xingyu Gao, ${ }^{d}$ Feng Gao, ${ }^{c}$ \\ Yingping Zou ${ }^{\mathrm{b}}$ and Shu Kong So*a \\ Correction for 'A disorder-free conformation boosts phonon and charge transfer in an electron-deficient- \\ core-based non-fullerene acceptor' by Chujun Zhang et al., J. Mater. Chem. A, 2020, 8, 8566-8574, DOI: \\ 10.1039/d0ta01260b
}

The authors regret that two of the author names, Zhenhuang Su and Xingyu Gao, are not displayed correctly in the published article. The corrected list of authors for this paper is as shown here.

The Royal Society of Chemistry apologises for these errors and any consequent inconvenience to authors and readers.

${ }^{a}$ Department of Physics, Institute of Advanced Materials, Hong Kong Baptist University, Kowloon Tong, Hong Kong SAR, P. R. China. E-mail: skso@hkbu.edu.hk ${ }^{b}$ College of Chemistry and Chemical Engineering, Central South University, Changsha 410083, Hunan, P. R. China

${ }^{c}$ Department of Physics, Chemistry and Biology (IFM), Linköping University, Linköping 58183, Sweden

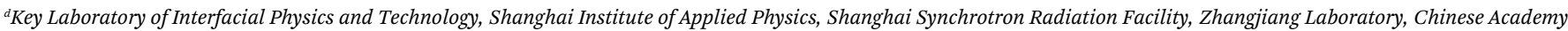
of Sciences, Shanghai 201204, China

$\dagger$ These authors contributed equally. 